\title{
Spectral function of graphene with short-range impurity centers
}

\author{
Yu.V. Skrypnyk \\ G.V. Kurdyumov Institute of Metal Physics of the National Academy of Sciences of Ukraine \\ 36 Vernadsky Ave., Kyiv 03680, Ukraine \\ V.M. Loktev \\ Bogolyubov Institute for Theoretical Physics of the National Academy of Sciences of Ukraine \\ 14-b Metrolohichna Str., Kyiv 03680, Ukraine \\ E-mail: vloktev@bitp.kiev.ua \\ Received May 20, 2008
}

\begin{abstract}
Spectral function of graphene with point substitutional defects is calculated for different impurity concentrations. It is demonstrated that features in the spectral function of graphene observed in ARPES experiments can be caused by the presence of a well-defined resonance state and are clearly pronounced at the impurity concentrations, which are of the order of the critical concentration for the impurity induced spectrum rearrangement.
\end{abstract}

PACS: 71.23.-k Electronic structure of disordered solids;

71.55.-i Impurity and defect levels;

81.05.Uw Carbon, diamond, graphite.

Keywords: spectral function of graphene, ARPES experiments, spectrum rearrangement.

\section{Introduction}

The most fascinating feature of graphene [1-3] is, indeed, the Dirac dispersion of charge carriers in the vicinity of the Fermi level. The linear dispersion of electrons is quite unusual for the condensed matter physics, and, in addition, it is realized in the case of graphene in a purely two-dimensional system. This linear dispersion leads to a number of challenging physical consequences, which attracted a considerable amount of scientific attention recently. If we are allowed to exaggerate a bit, than the Dirac dispersion in graphene is, probably, solely responsible for a constantly increasing amount of scientific research devoted to this material in the past years. Probably, it is permissible to say that this change in the wave vector power in the dispersion law did spawn an avalanche of scientific publications.

The Dirac dispersion of electrons in graphene, being initially indirectly confirmed by the unconventional quantum Hall effect, has been then directly observed in the ARPES experiments [4,5]. It has been shown that even a simple tight-binding model for the one-atom thick layer of carbon atoms can be successfully used to fit the obtained experimental data on the dispersion law in graphene. Along with that, a clear sign of the impurity resonance has been reported [5]. It has been concluded that defect-induced localized or quasi-localized states can contribute significantly to the density of states in graphene in the vicinity of the Dirac point in the spectrum.

In further ARPES measurements for graphene, potassium atoms were purposely deposited on it to dope the system with carriers and, thus, to shift the position of the Fermi level up from the Dirac point [6,7]. These experiments revealed that the spectral function does possess some intriguing features. It has been found that linear extrapolations of the dispersion curves from the valence band do not pass through the correspondent dispersion curves from the conduction band, and thus, there is no linear dispersion in a strict sense and Dirac cones are distorted. Apart from the overall shift of graphene bands to higher binding energies, the potassium doping leads to the appearance of a noticeable kink in the dispersion curves above the Dirac point. Its location in the energy axis does not change significantly with increasing the amount of deposited atoms. 
The origin of this kink has beet attributed to the electron-phonon coupling to the graphene in-plane optical vibrations. At the same time, the impurity scattering has been neglected. It was expected that the presence of impurity centers can only provide for a uniform background scattering rate $[6,7]$. However, the strength of the required electron-phonon coupling should be around five times larger than what could be expected to support this mechanism of kink formation. As an alternative, a theory for the phonon-mediated electron-electron interaction in graphene has been proposed [8]. The calculated spectral function showed that the electron-phonon coupling has a large effect on the band structure renormalization, but the resulting kink on the dispersion curve appeared to be much sharper than experimentally observed.

Another approach to the explanation of the spectral function in graphene has been undertaken in [9]. By means of the density functional theory calculations the electronic structure of graphene doped by the deposition of $\mathrm{Ca}$ atoms has been studied. Namely, the case of a large amount of foreign atoms, which reside in the $\mathrm{CaC}_{6}$ stoichiometry, has been considered. Authors found that, as the result of doping, the band structure of graphene is substantially affected, $\mathrm{Ca}$ bands occurs near the Fermi level and their hybridization with the states of bare graphene generates a strong non-linearity in the bands. They supposed that this non-linearity instead of many-body effects is revealed in ARPES experiments.

In our former studies $[10,11]$ we have demonstrated that substitutional impurities can lead to the appearance of resonance states near the Dirac point in the graphene's spectrum. The position of the resonance peak does not shift much with increasing the concentration of impurities [12]. Thus, it is enticing to suppose that the kink on the spectral function of graphene, which position also remains stable, can originate from the impurity resonance. It has been shown $[10,11]$ that even small amount of impurities with strong scattering potentials can cause the spectrum rearrangement in the electron subsystem of graphene. Below, we are describing the concentrational dynamics of the spectral function of graphene with the aim to illustrate how the process of the spectrum rearrangement is reflected in the spectral function features.

\section{Model of massless Dirac fermions}

The most simple and widely accepted way to model the electron dispersion in graphene in the vicinity of the intrinsic Fermi level is to use the picture of massless Dirac fermions. Of course, the linear dispersion domain in a strict sense spans for just several percents of the entire bandwidth, but it is the domain in which all the intriguing physical effects are acting out. In contrast to the two actual Dirac cones in the graphene's Brillouin zone, only a single Dirac cone is present in this straightforward ap- proach . Thus, the host Hamiltonian $\mathcal{H}_{0}$, which models the linear portion of the spectrum in graphene, reads

$$
\begin{gathered}
\mathcal{H}_{0}=\sum_{\mathbf{k}}\left[f(\mathbf{k}) c_{1}^{\dagger}(\mathbf{k}) c_{2}(\mathbf{k})+f^{*}(\mathbf{k}) c_{2}^{\dagger}(\mathbf{k}) c_{1}(\mathbf{k})\right], \\
c_{\alpha}(\mathbf{k})=\frac{1}{\sqrt{N}} \sum_{\mathbf{n}} \mathrm{e}^{i \mathbf{k n}} c_{\mathbf{n} \alpha}, \quad f(\mathbf{k})=\frac{a}{2 \sqrt{\pi}}\left(k_{x}+i k_{y}\right),
\end{gathered}
$$

where $a$ is a lattice constant, $\mathbf{n}$ runs over lattice cells, $\alpha$ enumerates the two required sublattices, $c_{\mathbf{n} \alpha}^{\dagger}$ and $c_{\mathbf{n} \alpha}$ are the electron creation and annihilation operators on the respective lattice cites, and the magnitude of the hopping parameter is chosen so that the resulting bandwidth is unity, when the Brillouin zone is approximated with a circle. Obviously, the dispersion relation, which follows from the chosen Hamiltonian (1), has the desired Dirac appearance,

$$
\varepsilon(\mathbf{k})= \pm \frac{a}{2 \sqrt{\pi}} k,
$$

throughout the entire circular Brillouin zone. In order to get a better feeling of the physical essence of the massless Dirac fermion model for graphene, it seems worthwhile to calculate within it the host single-electron Green's function

$$
\mathbf{g}=\left(\varepsilon-\mathcal{H}_{0}\right)^{-1}
$$

in the cite representation directly. The Green's function matrix elements between the cites on the same sublattice are given by

$$
\begin{aligned}
g_{0 \alpha \mathbf{n} \alpha}=\frac{a^{2}}{(2 \pi)^{2}} \varepsilon & \int_{0}^{\frac{2 \sqrt{\pi}}{a}} \frac{k d k}{\varepsilon^{2}-\left(\frac{a}{2 \sqrt{\pi}} k\right)^{2}} \int_{0}^{2 \pi} \exp (i k n \cos \phi) d \phi= \\
& =2 \varepsilon \int_{0}^{1} \frac{J_{0}(2 \sqrt{\pi} t r)}{\varepsilon^{2}-t^{2}} t d t
\end{aligned}
$$

where $r=n / a$, and $J_{0}$ is the zeroth order Bessel function of the first kind. Immediately, for the diagonal element of the Green's function one has:

$$
g_{\mathbf{n} \alpha \mathbf{n} \alpha} \equiv g_{0}=\varepsilon \int_{0}^{1} \frac{d x}{\varepsilon^{2}-x}=\varepsilon \ln \frac{\varepsilon^{2}}{\varepsilon^{2}-1}, \quad|\varepsilon|>1,
$$

and, respectively,

$$
g_{0}=\varepsilon\left(\ln \frac{\varepsilon^{2}}{1-\varepsilon^{2}}-i \pi \operatorname{sgn}(\varepsilon)\right), \quad|\varepsilon|<1 .
$$

Similarly, the magnitude of the imaginary part of the Green's function matrix element between two separate sites can be obtained without excessive efforts, 


$$
\begin{gathered}
\operatorname{Im} g_{0 \alpha \mathbf{n} \alpha}=-\pi \int_{0}^{1}[\delta(\varepsilon-t)+\delta(\varepsilon+t)] J_{0}(2 \sqrt{\pi} t r) t d t= \\
=-\pi|\varepsilon| J_{0}(2 \sqrt{\pi}|\varepsilon| r) .
\end{gathered}
$$

When calculating the real part of this matrix element, it is convenient to resort to an obvious approximation and extend the integration to infinity,

$$
\operatorname{Re} g_{0 \alpha \mathbf{n} \alpha} \approx 2 \varepsilon \text { V.P. } \int_{0}^{\infty} \frac{J_{0}(2 \sqrt{\pi} t r)}{\varepsilon^{2}-t^{2}} t d t=\pi \varepsilon Y_{0}(2 \sqrt{\pi}|\varepsilon| r)
$$

where $Y_{0}$ is the zeroth order Bessel function of the second kind.

Indeed, the obtained expression for the real part of the matrix element should correspond to (6) at $r=0$. However, the Bessel function of the second kind does diverge when its argument approaches zero. Thus, it is obvious that (8) is not valid at small distances $r$, which is a consequence of the adopted approximation. Nevertheless, it is not difficult to verify that this approximation works fairly well at small $\varepsilon$ for intersite distances $r$ starting from just several units in magnitude. Combining the above results, we get:

$$
g_{0 \alpha \mathbf{n} \alpha} \approx \pi \varepsilon\left[Y_{0}(2 \sqrt{\pi}|\varepsilon| r)-i \operatorname{sgn}(\varepsilon) J_{0}(2 \sqrt{\pi}|\varepsilon| r)\right] .
$$

The matrix elements between sites located in different sublattices can be written in the same way,

$$
\begin{gathered}
g_{0 \alpha \mathbf{n} \beta}=\frac{a^{2}}{(2 \pi)^{2}} \int_{0}^{\frac{2 \sqrt{\pi}}{a}} \frac{\frac{a}{2 \sqrt{\pi}}}{\varepsilon^{2}-\left(\frac{a}{2 \sqrt{\pi}} k\right)^{2}} k^{2} d k \mathrm{e}^{i \phi^{\prime}} \times \\
\quad \times \int_{0}^{2 \pi} \exp \left(i\left[\phi-\phi^{\prime}+k n \cos \left(\phi-\phi^{\prime}\right)\right]\right) d \phi,
\end{gathered}
$$

where $\beta \neq \alpha$ (cp. (4)), and $\phi^{\prime}$ is the polar angle of the radius-vector $\mathbf{n}$. Since

$$
\int_{0}^{2 \pi} \exp \left(i\left[\phi-\phi^{\prime}+k n \cos \left(\phi-\phi^{\prime}\right)\right]\right) d \phi=i 2 \pi J_{1}(k n),
$$

where $J_{1}$ is the Bessel function of the first kind and the order unity, the expression for the matrix element in question can be simplified,

$$
\begin{aligned}
g_{0 \alpha \mathbf{n} \beta}= & \frac{a^{2} i \mathrm{e}^{i \phi^{\prime}}}{2 \pi} \int_{0}^{\frac{2 \sqrt{\pi}}{a}} \frac{\frac{a}{2 \sqrt{\pi}} J_{1}(k n)}{\varepsilon^{2}-\left(\frac{a}{2 \sqrt{\pi}} k\right)^{2}} k^{2} d k= \\
& =2 i \mathrm{e}^{i \phi^{\prime}} \int_{0}^{1} \frac{J_{1}(2 \sqrt{\pi} t r)}{\varepsilon^{2}-t^{2}} t^{2} d t .
\end{aligned}
$$

Taking the imaginary part of the integral in (12) yields:

$$
\begin{gathered}
2 \operatorname{Im}\left[\int_{0}^{1} \frac{J_{1}(2 \sqrt{\pi} t r)}{\varepsilon^{2}-t^{2}} t^{2} d t\right]= \\
=-\frac{\pi}{\varepsilon} \int_{0}^{1} J_{1}(2 \sqrt{\pi} t r)[\delta(\varepsilon-t)+\delta(\varepsilon+t)] t^{2} d t= \\
=-\pi \varepsilon J_{1}(2 \sqrt{\pi}|\varepsilon| r) .
\end{gathered}
$$

As before, by extending the upper limit of the integral in (12) to infinity when calculating its real part, we obtain,

$$
\begin{gathered}
2 \operatorname{Re}\left[\int_{0}^{1} \frac{J_{1}(2 \sqrt{\pi} t r)}{\varepsilon^{2}-t^{2}} t^{2} d t\right] \approx 2 \text { V.P. } \int_{0}^{\infty} \frac{J_{1}(2 \sqrt{\pi} t r)}{\varepsilon^{2}-t^{2}} t^{2} d t= \\
=\pi|\varepsilon| Y_{1}(2 \sqrt{\pi}|\varepsilon| r)
\end{gathered}
$$

where reservations similar to the ones for (8) should be kept in mind at the small intersite distances $r$. Finally, the matrix element of the host Green's function between sites on different sublattices can be expressed as

$$
g_{0 \alpha \mathbf{n} \beta} \approx i \pi \mathrm{e}^{i \phi^{\prime}} \varepsilon\left[\operatorname{sgn}(\varepsilon) Y_{1}(2 \sqrt{\pi}|\varepsilon| r)-i J_{1}(2 \sqrt{\pi}|\varepsilon| r)\right] .
$$

It should be stressed upon the fact that all matrix elements of the host Green's function are vanishing to zero when energy is approaching the Dirac point in the adopted model of massless charge carriers for graphene.

\section{Substitutional impurities}

In order to take into account the effect of impurities on the spectral function in graphene, it is supposed that they are substitutional and distributed absolutely at random in the crystal lattice with some concentration $c$. In addition, it is postulated that the presence of an impurity at a given lattice site leads only to a change of the corresponding on-site energy in the tight-binding Hamiltonian of the system. In other words, impurities are treated within the conventional framework of a substitutional binary alloy with a diagonal disorder. The adopted approach is frequently referred to as the Lifshitz impurity model for historical reasons [13]. The impurity perturbation is often chosen in the asymmetric way, that is, the on-site potentials at the sites occupied by impurities are taken to be equal to some fixed value $V_{L}$, while at those lattice sites, which remain to be occupied by host atoms, the on-site potentials are taken to be equal to zero. The corresponding Hamiltonian of the system with impurities reads,

$$
\mathcal{H}=\mathcal{H}_{0}+\mathcal{H}_{\text {imp }}, \mathcal{H}_{\text {imp }}=V_{L} \sum_{\mathbf{n}, \alpha}^{\prime} c_{\mathbf{n} \alpha}^{\dagger} c_{\mathbf{n} \alpha}
$$


where summation is restricted only to those sites, which are occupied by impurities. The self-energy $\Sigma$, which is defined by the Dyson equation,

$$
\mathbf{G}=\mathbf{g}+\mathbf{g} \Sigma \mathbf{G},
$$

where $\mathbf{G}$ is the Green's function of the disordered system averaged over different impurity distributions,

$$
\mathbf{G}=\left\langle(\varepsilon-\mathcal{H})^{-1}\right\rangle,
$$

can be expanded into a series according to the size of the cluster of indirectly interacting impurity centers,

$$
\Sigma_{\alpha \beta}(\mathbf{k})=\delta_{\alpha \beta} \sigma^{(1)}+\sigma_{\alpha \beta}^{(2)}(\mathbf{k})+\ldots,
$$

because the translational invariance is restored by the procedure of the configurational averaging $[14,15]$. Here the first momentum-independent term $\sigma^{(1)}$ is the familiar coherent potential approximation for the self-energy,

$$
\sigma^{(1)}=\frac{c V_{L}}{1-\left(V_{L}-\sigma^{(1)}\right) g_{0}\left(\varepsilon-\sigma^{(1)}\right)},
$$

with its inherent self-consistency. The second term, $\sigma_{\alpha \beta}^{(2)}(\mathbf{k})$ represents the contribution from all possible scatterings on the pair of impurities,

$$
\begin{aligned}
\sigma_{\alpha \beta}^{(2)}(\mathbf{k}) & =\sum_{l, m} \xi_{l} \xi_{m}\left(\delta_{\alpha \beta} \sum_{\mathbf{n} \gamma \neq 0 \alpha} \frac{\tau_{l}^{3} \tau_{m}^{2} \mathbf{G}_{0 \alpha \mathbf{n} \gamma}^{2} \mathbf{G}_{\mathbf{n} \gamma 0 \alpha}^{2}}{1-\tau_{l} \tau_{m} \mathbf{G}_{0 \alpha \mathbf{n} \gamma} \mathbf{G}_{\mathbf{n} \gamma 0 \alpha}}+\right. \\
& \left.+\sum_{\mathbf{n} \beta \neq 0 \alpha} \frac{\tau_{l}^{2} \tau_{m}^{2} \mathbf{G}_{0 \alpha \mathbf{n} \beta}^{2} \mathbf{G}_{\mathbf{n} \beta 0 \alpha}}{1-\tau_{l} \tau_{m} \mathbf{G}_{0 \alpha \mathbf{n} \beta} \mathbf{G}_{\mathbf{n} \beta 0 \alpha}} \mathrm{e}^{i \mathbf{k n}}\right)
\end{aligned}
$$

where $\tau_{l}$ denotes the single-site $T$-matrix,

$$
\tau_{l}=\frac{V_{l}-\sigma^{(1)}}{1-\left(V_{l}-\sigma^{(1)}\right) g_{0}\left(\varepsilon-\sigma^{(1)}\right)},
$$

while indices $l$ and $m$ enumerate two atom types of the disordered binary alloy. Respective to the magnitude of this index, the factor $\xi_{l}$ is either equal to the impurity concentration $c$, which corresponds to the sites occupied by defects, or to $1-c$, which corresponds to the remaining host atoms. In concert, the variable $V_{l}$ attains the value $V_{L}$ or 0 . Contributions from clusters containing three or more impurities are omitted from (19).

The series (19) has been extensively analyzed for the disordered systems of different dimensionality and for a wide range of dispersion laws. This analysis leads to a conclusion that the series for the self-energy basically behaves as a geometric progression. For most disordered systems, the contribution from pair impurity clusters (21) should be compared to contributions from impurity clusters of larger size in order to get this result. The common ratio of this progression,

$$
R(\varepsilon)=\sum_{l} \xi_{l} \tau_{l}^{2} \sum_{\mathbf{n} \beta \neq 0 \alpha} \mathbf{G}_{0 \alpha \mathbf{n} \beta}^{2},
$$

is the small parameter of the self-energy expansion into the groups of impurity centers, which simultaneously plays a role of the coherent potential approximation validity criterion and is, indeed, an essential parameter of the disordered system.

It is expected that there is a strong correlation between the magnitude of this small parameter and the localization of the states in the disordered system. For the energies approaching any of the van Hove singularities in the host system or the strong impurity resonance the magnitude of this small parameter is increasing, which indicates an increase in scatterings by impurity clusters. In contrast, all the cluster terms can be omitted from the self-energy expansion, when the inequality

$$
|R(\varepsilon)| \leq 1 / 2
$$

holds and energy falls within the continuous host spectrum [16]. Then, only the first term corresponding to the coherent potential approximation can be retained in the series. Thus, this condition outlines those portions of the spectrum, in which the coherent potential approximation is valid. In addition, there is a strong evidence that states in the disordered system are strongly localized, when the inequality (24) is violated. Therefore, generally speaking, the magnitude of this parameter should be carefully monitored, especially inside the most dangerous spectral domains located around van Hove singularities and impurity resonances, as was mentioned above.

Lets assume that the condition (24) is fulfilled. Then, the self-energy is given by the first term of the series (19) with a sufficient accuracy. At a small $(c<<1)$, but still finite concentration of impurities, the multiple-occupancy corrections that are embedded into the coherent potential approximation are also insignificant. As a result, a selfconsistent expression for the self-energy can be further simplified to the so-called modified propagator method,

$$
\Sigma \approx \sigma \mathbf{I}, \quad \sigma=\frac{c V_{L}}{1-V_{L} g_{0}(\varepsilon-\sigma)}, \quad c<<1 .
$$

Since we are concerned only with the close vicinity of the Dirac point in graphene's spectrum, the obtained expression for the diagonal element of the host Green's function can be simplified too,

$$
g_{0}(\varepsilon) \approx 2 \varepsilon \ln |\varepsilon|-i \pi|\varepsilon|, \quad|\varepsilon|<<1 .
$$

By utilizing the standard substitution,

$$
\varepsilon-\sigma=\kappa \exp (i \varphi), \quad \kappa>0, \quad 0<\varphi<\pi,
$$

and taking into account the approximate expression for the diagonal element of the Green's function (26), the 
imaginary part of the self -consistency condition in (26) can be written as follows $[10,11]$,

$$
\begin{gathered}
c V_{L}^{2}[2 \ln \kappa+(2 \varphi-\pi) \cot \varphi]+ \\
+\left[1-V_{L} \kappa(2 \ln \kappa \cos \varphi-(2 \varphi-\pi) \sin \varphi)\right]^{2}+ \\
+\left[V_{L} \kappa(2 \ln \kappa \sin \varphi+(2 \varphi-\pi) \cos \varphi)\right]^{2}=0 .
\end{gathered}
$$

This equation has always two solutions for the phase of the renormalized energy $\varphi$, when its modulus $\kappa$ exceeds a certain threshold value, provided that the strength of the impurity perturbation $V_{L}$ and the impurity concentration $c$ are given. Then, the corresponding energies energies can be obtained from the real part of (25),

$$
\varepsilon=\frac{\kappa \cos \varphi+c V_{L}\left[1-V_{L} \kappa(2 \ln \kappa \cos \varphi-(2 \varphi-\pi) \sin \varphi)\right]}{\left[1-V_{L} \kappa(2 \ln \kappa \cos \varphi-(2 \varphi-\pi) \sin \varphi)\right]^{2}+\left[V_{L} \kappa(2 \ln \kappa \sin \varphi+(2 \varphi-\pi) \cos \varphi)\right]^{2}} .
$$

These two equations, (28) and (29) allow to establish the implicit relation between the renormalized energy $\varepsilon-\sigma$ and the energy of the charge carrier $\varepsilon$, which, in a turn, provides the energy dependence of the self-energy.

\section{Spectral function}

According to the reasoned above approximation (25), the self-energy is indeed site-diagonal. It is not difficult to make sure that it is not only diagonal in sublattice indices, but also is identical on both sublattices. Therefore, after the summation over sublattice indices, the spectral function of graphene with impurities, which can be obtained by the ARPES measurements, takes the form:

$$
A(\mathbf{k}, \varepsilon) \approx \frac{2}{\pi} \operatorname{Im} \frac{\varepsilon-\sigma}{(\varepsilon-\sigma)^{2}-\left(\frac{a}{2 \sqrt{\pi}} k\right)^{2}} .
$$

Below, for simplicity, we will assume that the unit length is chosen in such a way that $a=2 \sqrt{\pi}$.
In the former study $[10,11]$, it has been demonstrated that there is a certain critical concentration of impurities $c_{0} \sim 1 / V_{L}^{2}$ determined by the strength of the impurity perturbation, at which spectral properties of the disordered system described by the model of massless Dirac fermions undergo a dramatical qualitative change, or, in other words, the spectrum rearrangement takes place. This critical concentration has been related to a spacial overlap between individual impurity states. Below we illustrate those changes that occur in the spectral function (30) with increasing the concentration of substitutional impurities. The concentrational dynamics of the spectral function for the relatively weak impurity perturbation $V_{L}=-2$ is shown in Figs. 1,2. The viewpoint is located on the side of the conduction band to make figures more expository.

According to earlier estimations [10,11], the corresponding critical concentration $c_{0}$ at $V_{L}=-2$ is around 0.01 . Impurities characterized by this potential, which is comparable to the bandwidth in magnitude, does not lead to the appearance of any well-defined resonance in the

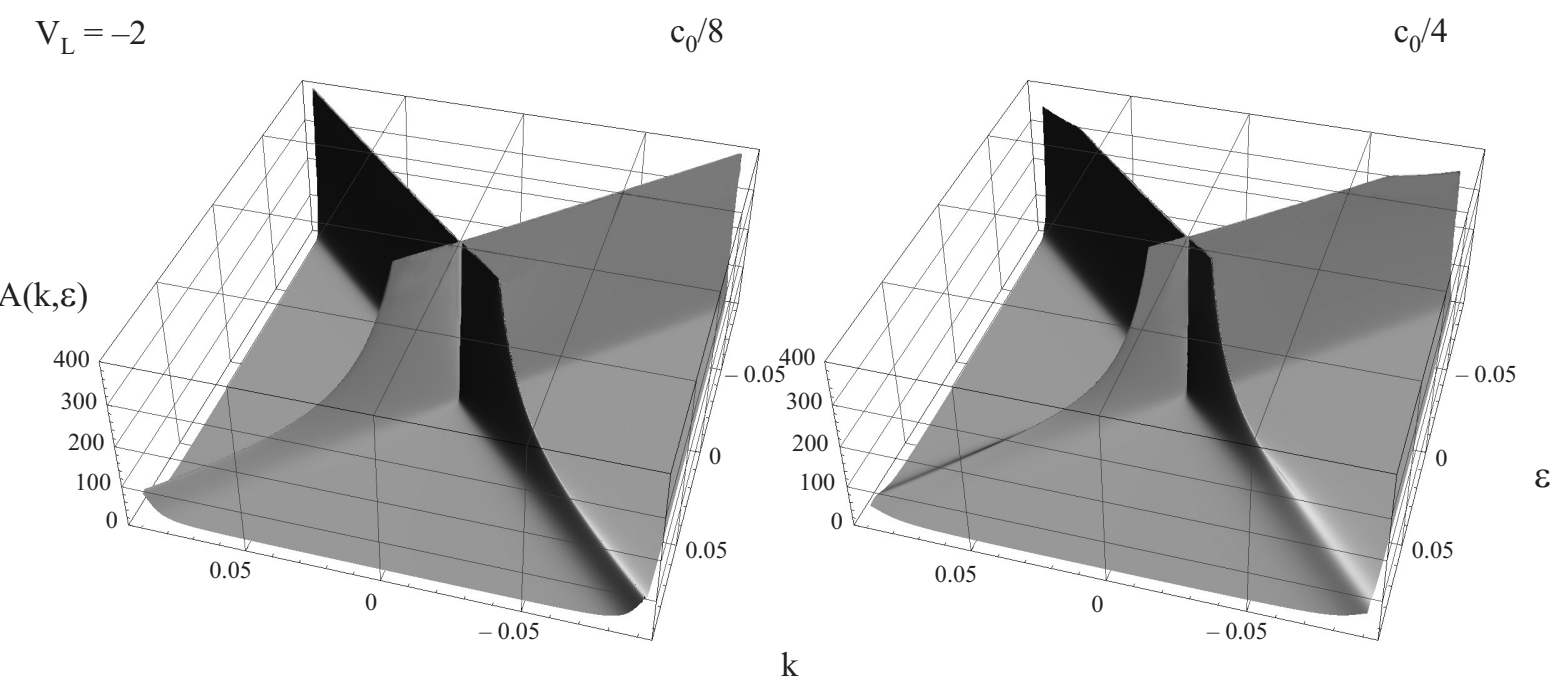

Fig. 1. Spectral function of graphene for $V_{L}=-2$ at $c=c_{0} / 8$ and $c=c_{0} / 4, c_{0}=0.012$. 


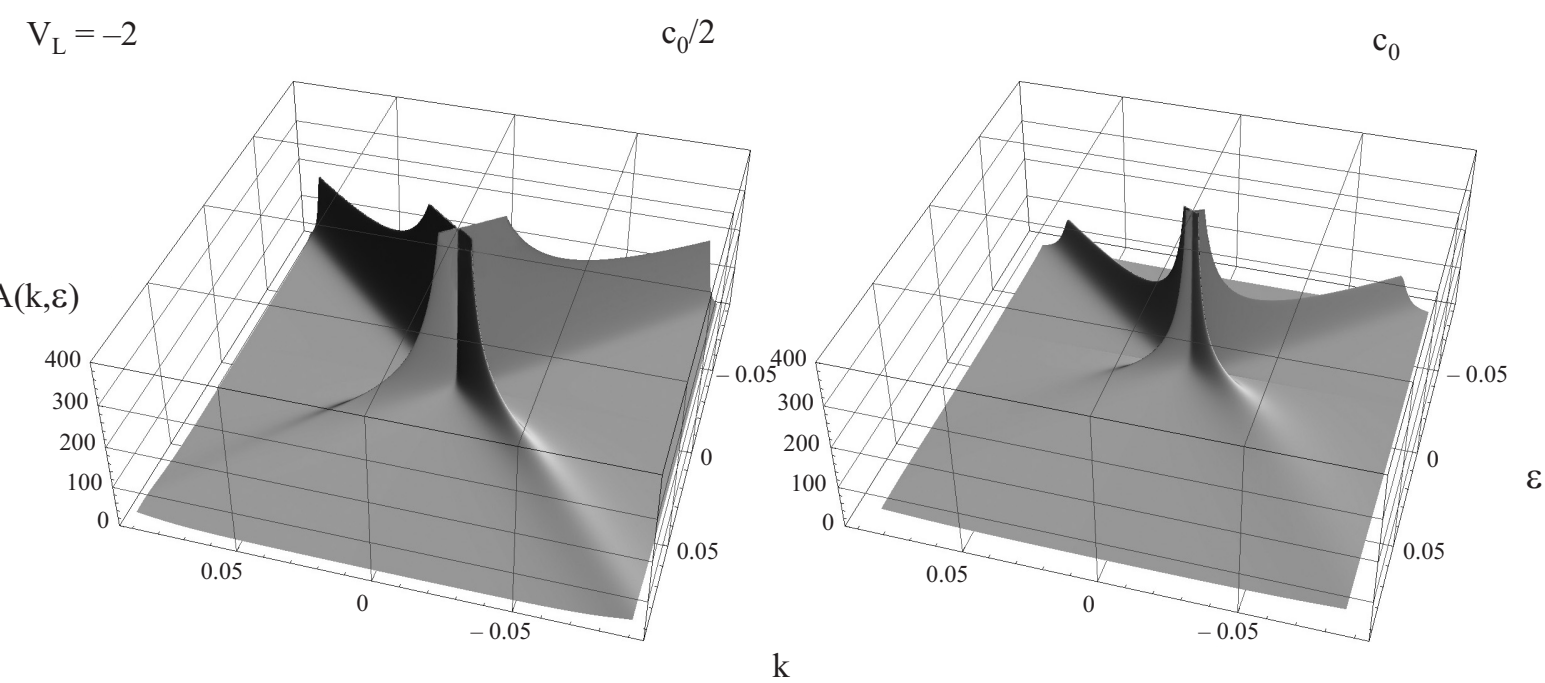

Fig. 2. Spectral function of graphene for $V_{L}=-2$ at $c=c_{0} / 2$ and $c=c_{0}, c_{0}=0.012$.

spectrum. This explains the smooth and monotonic behavior of the spectral function in Figs. 1,2. Nevertheless, these figures clearly show that the initial symmetry between the valence band and the conduction band in graphene is violated under the presence of impurities. Apart from the shift in the Dirac point position towards negative energies, it is evident that states in the conduction band are significantly more damped due to the scatterings on impurity centers. The damping is gradually increasing with an increase in the impurity concentration. This increase of the damping, in a turn, smears maximums of the spectral function and reduces their heights. In the low concentration regime with respect to the onset of the spectrum rearrangement $\left(c<c_{0}\right)$ there should be present a so-called concentration smearing region around the Dirac point $[10,11]$. In this region the condition (24) is not met, the states in the disordered system are localized and the approach, outlined above for the self-energy calculation, does fail. However, the width of this region at $c<c_{0}$ is exponentially small compared to the bandwidth, and thus it will not be discernible in the figures.

It should be noted that the case of $V_{L}=-4$ is in some sense marginal. The respective concentrational dynamics of the spectral function is presented in Figs. 3,4.

In this case the critical concentration of the spectrum rearrangement $c_{0}$ for $V_{L}=-4$ is significantly less than for $V_{L}=-2$ and amounts to approximately 0.002 . The shift in the Dirac point position is less articulated, albeit it is clearly expressed. Impurity perturbation of this strength leads to the appearance of the not so prominent resonance state in the conduction band. The presence of this smeared resonance explains the sags in the ridges of the spectral

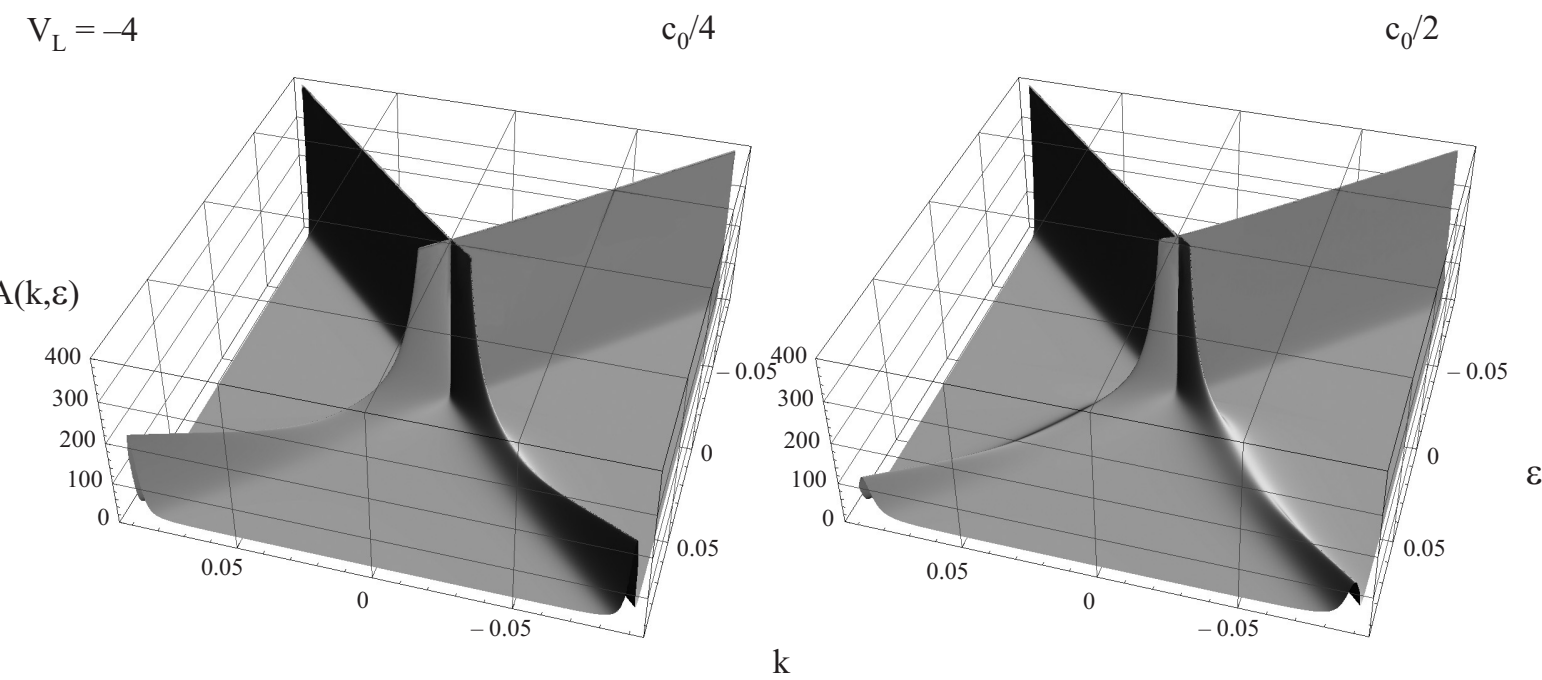

Fig. 3. Spectral function of graphene for $V_{L}=-4$ at $c=c_{0} / 4$ and $c=c_{0} / 2, c_{0}=0.00243$. 


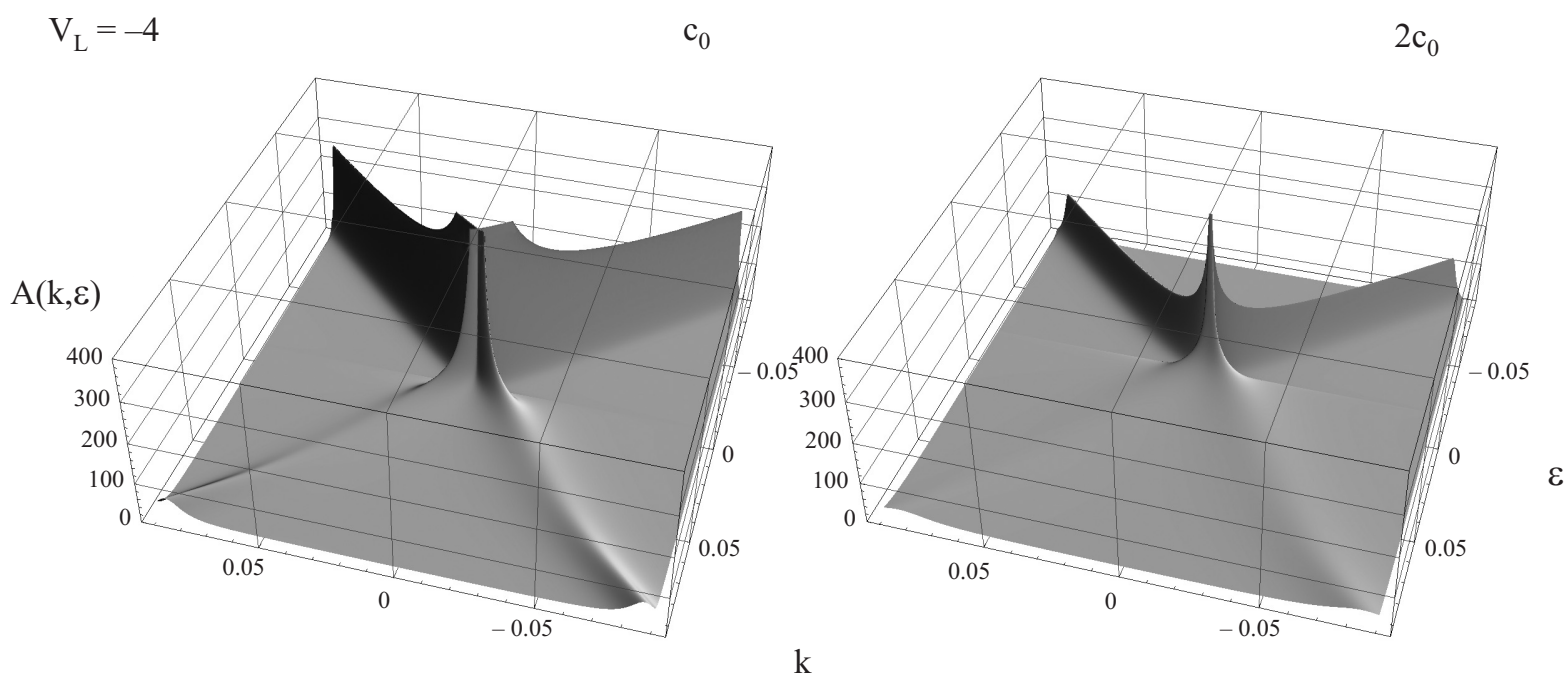

Fig. 4. Spectral function of graphene for $V_{L}=-4$ at $c=c_{0}$ and $c=2 c_{0}, c_{0}=0.00243$.

function, which are distinctly seen at $c=c_{0} / 4$. These sags nearly totally disappear at higher impurity concentrations, so that the overall picture closely resembles the former case and is characterized by the marked asymmetry between the valence band and the conduction band in the amount of damping. As had been demonstrated in $[10,11]$, the width of the concentration smearing region grows rapidly with an increase in the impurity concentration approximately proportional to $\sqrt{c}$, when the impurity concentration exceeds the critical value $c_{0}$. While, strictly speaking, our approach to the spectral function calculation is not applicable for the energies belonging to this region, the result for $c=2 c_{0}$ is also provided. Doing this, we keep in mind that withing the concentration smearing region states are anyway highly damped, and thus the spectral function is already smeared out and almost featureless.
The evolution of the spectral function with increasing the impurity concentration for the strong impurity perturbation $V_{L}=-8$ exhibits the most intense variations. Corresponding graphs are depicted in Figs. 5,6.

When the impurity perturbation is that strong, there is a well-defined resonance state not far from the Dirac point. Its energy $\varepsilon_{r}$ can be obtained from the Lifshitz equation:

$$
1=V_{L} \operatorname{Re} g_{0}\left(\varepsilon_{r}\right) \approx 2 V_{L} \varepsilon_{r} \ln \left|\varepsilon_{r}\right| .
$$

As a consequence, the sags in the ridges of the spectral function, which are located around the resonance energy, are sharply incised and remain manifested at the impurity concentrations of the order of $c_{0}$. To emphasize this fact, we presented the spectral function at impurity concentrations exceeding the critical one, even though, at these concentrations there is a noticeable region of concentration smearing around the resonance energy, where results

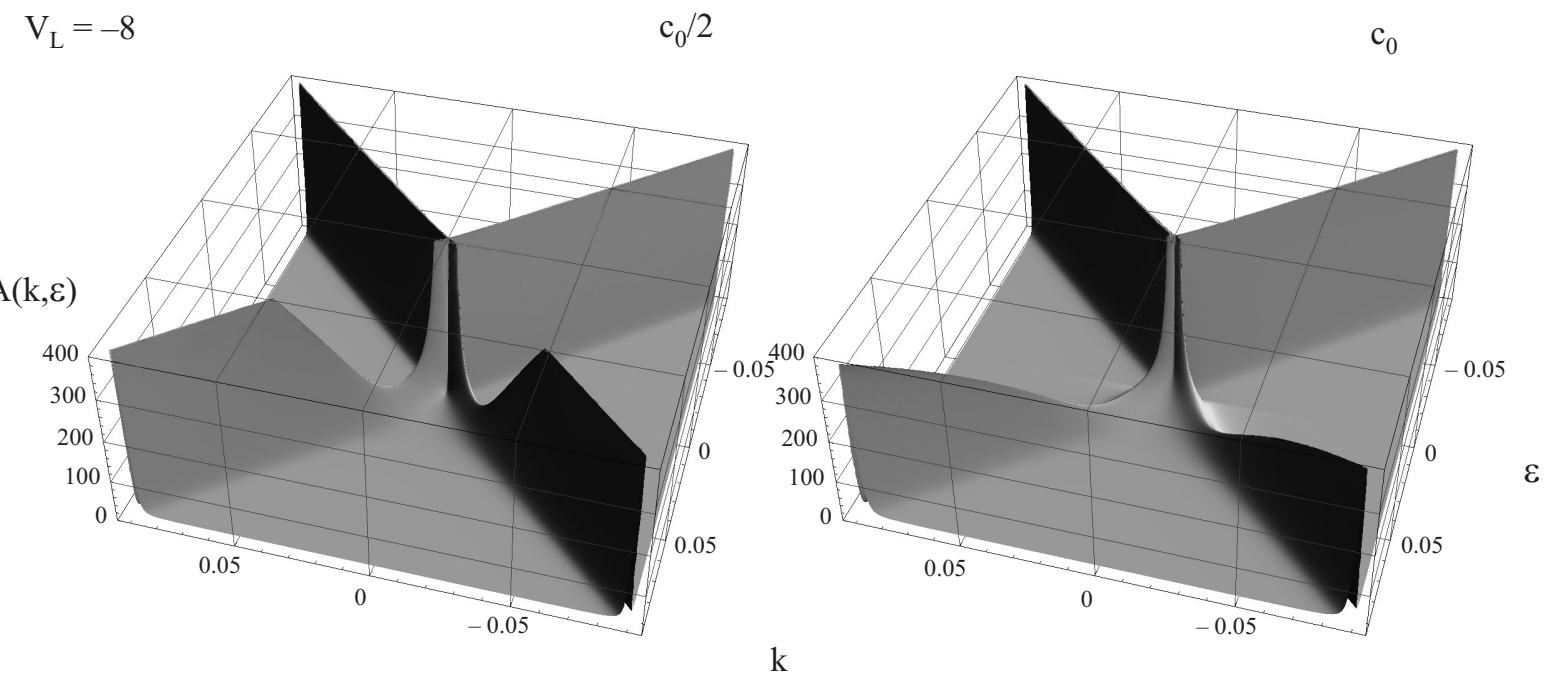

Fig. 5. Spectral function of graphene for $V_{L}=-8$ at $c=c_{0} / 2$ and $c=c_{0}, c_{0}=0.000523$. 


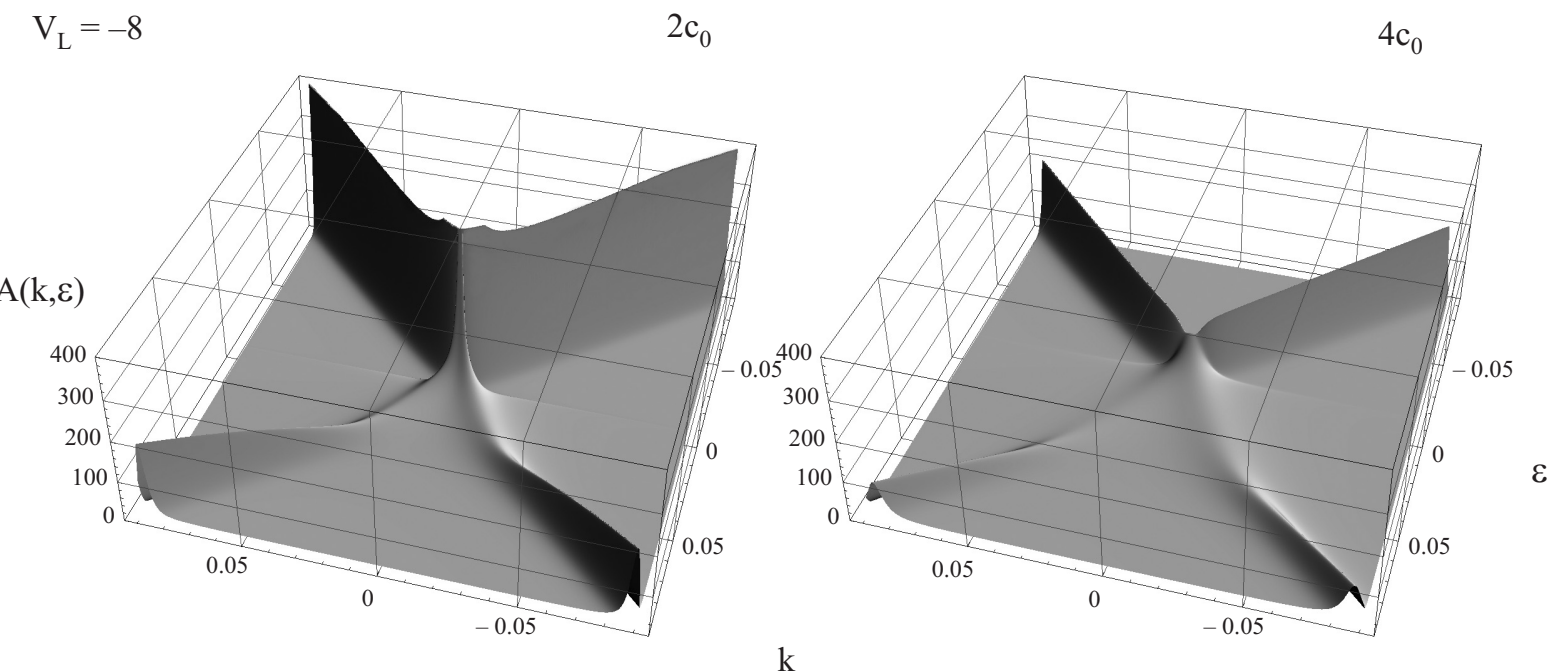

Fig. 6. Spectral function of graphene for $V_{L}=-8$ at $c=2 c_{0}$ and $c=4 c_{0}, c_{0}=0.000523$.

can not be fully justified (which has been already discussed above for the case $V_{L}=-4$ ). The width of each sag is gradually increasing with increasing the impurity perturbation.

The critical concentration for $V_{L}=-8$ is close to 0.0005 . Naturally, the shift of the Dirac point position has diminished. In contrast, the asymmetry between the conduction band and the valence band is more substantial. To make this asymmetry evident, density plots of the spectral function are given below in Figs. 7,8 for the same parameters as in Figs. 5,6.

In these figures darker shades of gray color correspond to the larger magnitudes of the spectral function and vice versa. Thin lines are provided as eye guides only and represent the unperturbed dispersion of electrons in graphene displaced so that they are crossing at the shifted position of the Dirac point. First of all, it is observable in these figures that the linear extrapolation of the dispersion lines in the valence band does not pass though the dispersion lines in the conduction band. Second, there is an apparent kink in the dispersion lines of the conduction band just above the shifted Dirac point in the spectrum. With increasing impurity concentration both these features are becoming more and more pronounced. Visually they are not different from the features in the spectral function obtained in ARPES experiments for graphene with deposited potassium atoms $[6,7]$.

\section{Conclusion}

We have shown the way in which the spectral function in graphene with point defects can be calculated by the
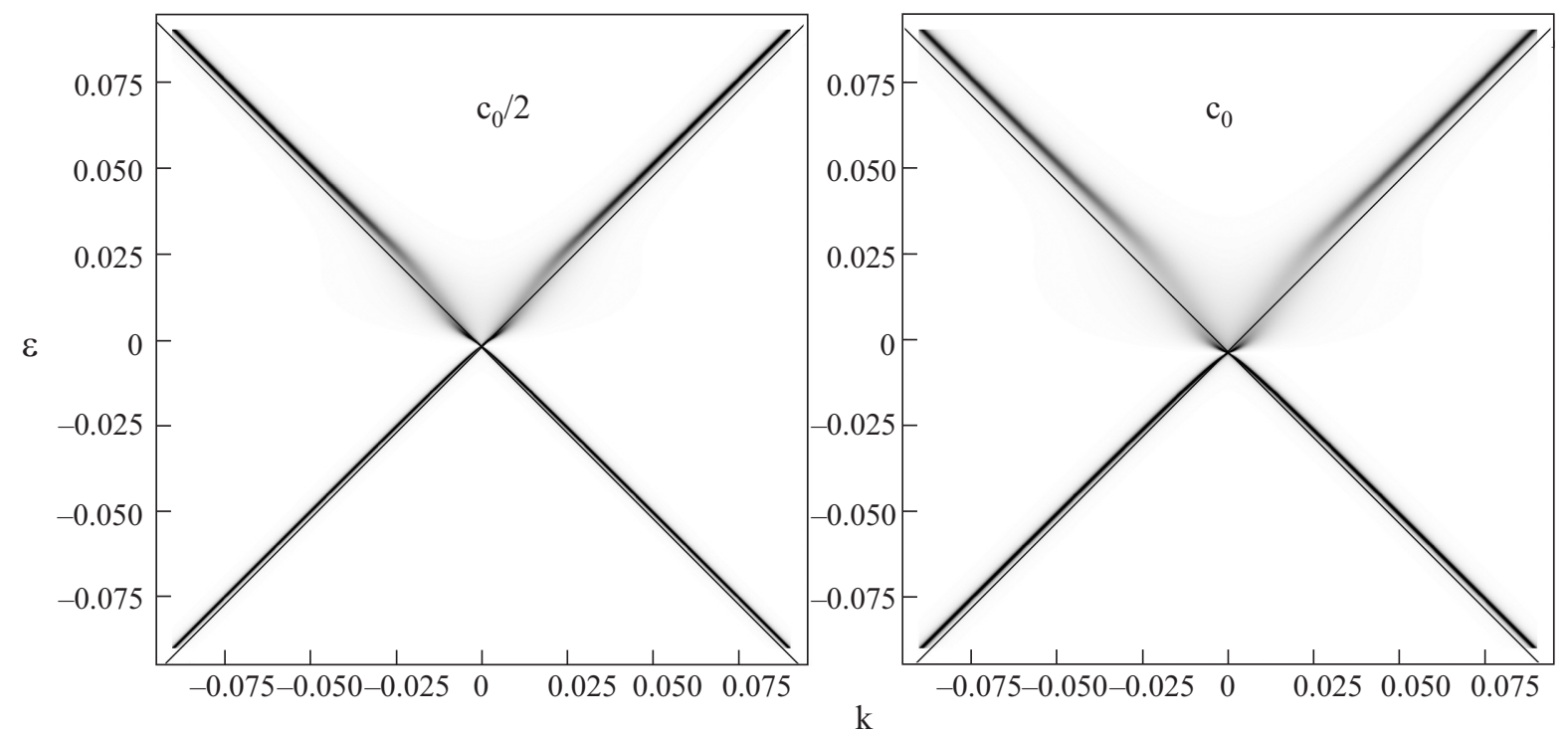

Fig. 7. Density plot of the spectral function of graphene for $V_{L}=-8$ at $c=c_{0} / 2$ and $c=c_{0}, c_{0}=0.000523$. 

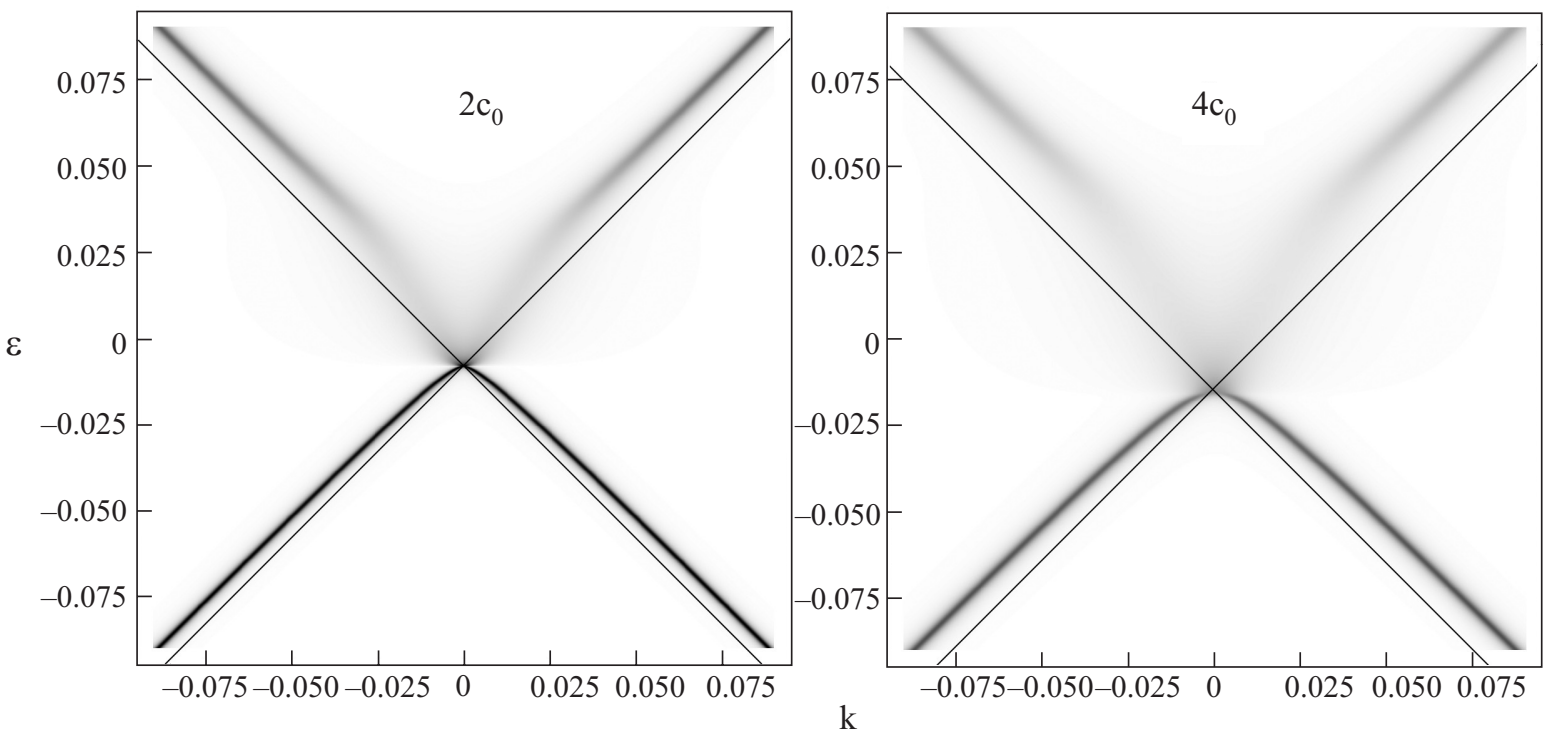

Fig. 8. Density plot of the spectral function of graphene for $V_{L}=-8$ at $c=2 c_{0}$ and $c=4 c_{0}, c_{0}=0.000523$.

method of the cluster expansion for the self energy. The evolution of the resulting spectral function with an increase in the impurity concentration has been discussed for a range of impurity perturbations varying in strength. With increasing the perturbation strength, the difference between the conduction band and the valence band is becoming more elaborated. Not only the damping of the extended states is considerably larger in the conduction band at the strong impurity perturbation (for the chosen sign of it), but also the extensions of the ridges in the spectral function for the valence band start to deviate from the ones for the conduction band in a more apparent way. In addition, when the impurity perturbation is strong enough to produce a well-defined resonance state above the Dirac point, the kink in the dispersion curve around the energy of this resonance is becoming clearly articulated. Thus, the model of point defects yields results that are at least qualitatively correspondent to the features of the spectral function seen in the ARPES measurements of graphene $[6,7]$. Since the impurity concentration has not been carefully monitored in $[6,7]$, it seems that it is too early to make any qualitative comparison between theoretical and experimental results. However, we conclude that impurity effects can be considered as a strong candidate for the explanation of the characteristic spectral function features in graphene and, for sure, can not be overlooked. The obtained results are also relevant in the case when vacancies play the role of impurity centers (for vacancies the impurity potential should be set to infinity).

This work has been partially supported by the Scientific Program Nanostructural Systems, Nanomaterials and Nanotechnologies of the National Academy of Sciences of Ukraine (grant No. 10/07-N).
1. K.S. Novoselov, A.K. Geim, S.V. Morozov, D. Jiang, M.I. Katsnelson, I.V. Grigorieva, S.V. Dubonos, and A.A. Firsov, Nature 438, 197 (2005).

2. Y. Zhang, Y.-W. Tan, H.L. Stormer, and P. Kim, Nature 438, 201 (2005).

3. A.K. Geim and K.S. Novoselov, Nature Mater. 6, 183 (2007).

4. S.Y. Zhou, G.-H. Gweon, C.D. Spataru, J. Graf, D.-H. Lee, Steven G. Louie, and A. Lanzara, Phys. Rev. B71, 161403(R) (2005).

5. S.Y. Zhou, G.-H. Gweon, J. Graf, A.V. Fedorov, C.D. Spataru, R.D. Diehl, Y. Kopelevich, D.-H. Lee, Steven G. Louie, and A. Lanzara, Nature Phys. 2, 595 (2006).

6. Aaron Bostwick, Taisuke Ohta, Thomas Seyller, K. Horn, and Eli Rotenberg, Nature Phys. 3, 36 (2007).

7. Jessica L. McChesney, Aaron Bostwick, Taisuke Ohta, Konstantin V. Emtsev, Thomas Seyller, Karsten Horn, and Eli Rotenberg, cond-mat/0705.3264 (unpublished).

8. Wang-Kong Tse and S. Das Sarma, Phys. Rev. Lett. 99, 236802 (2007).

9. Matteo Calandra and Francesco Mauri, Phys. Rev. B76, 161406(R) (2007).

10. Yu.V. Skrypnyk and V.M. Loktev, Phys. Rev. B73, 241402(R) (2006).

11. Yu.V. Skrypnyk and V.M. Loktev, Fiz. Nizk. Temp. 33, 1002 (2007) [Low Temp. Phys. 33, 762 (2007)].

12. Yu.V. Skrypnyk and V.M. Loktev, Phys. Rev. B75, 245401 (2007)

13. I.M. Lifshitz, Adv. Phys. 13, 483 (1964).

14. M.A. Ivanov, V.M. Loktev, and Yu.G. Pogorelov, Phys. Rep. 153, 209 (1987).

15. I.M. Lifshitz, S.A. Gredeskul, and L.A. Pastur, Introduction to the Theory of Disordered Systems, Wiley, N.Y. (1988).

16. Yu.V. Skrypnyk, Phys. Rev. B70, 212201 (2004). 